\title{
XMM-Newton observations of PSR J0726-2612, a radio-loud XDINS
}

\author{
Michela Rigoselli ${ }^{1,2}$, Sandro Mereghetti ${ }^{1}$, Valery Suleimanov ${ }^{3,4,5}$, Alexander Y. Potekhin ${ }^{6}$, Roberto Turolla ${ }^{7,8}$, \\ Roberto Taverna ${ }^{7,9}$, and Fabio Pintore ${ }^{1}$ \\ 1 INAF, Istituto di Astrofisica Spaziale e Fisica Cosmica Milano, Via A. Corti 12, 20133 Milano, Italy \\ 2 Dipartimento di Fisica G. Occhialini, Università degli Studi di Milano Bicocca, Piazza della Scienza 3, 20126 Milano, Italy \\ e-mail: m.rigoselli@campus.unimib.it \\ 3 Institut fur Astronomie und Astrophysik, Sand 1, 72076 Tubingen, Germany \\ ${ }^{4}$ Kazan (Volga region) Federal University, Kremlevskaja str., 18, Kazan 420008, Russia \\ 5 Space Research Institute of the Russian Academy of Sciences, Profsoyuznaya Str. 84/32, Moscow 117997, Russia \\ ${ }^{6}$ Ioffe Institute, Politekhnicheskaya 26, 194021 Saint Petersburg, Russia \\ 7 Dipartimento di Fisica e Astronomia, Università di Padova, Via F. Marzolo 8, 35131 Padova, Italy \\ 8 MSSL-UCL, Holmbury St. Mary, Dorking, Surrey RH5 6NT, UK \\ 9 Dipartimento di Matematica e Fisica, Università di Roma Tre, Via della Vasca Navale 84, 00146 Roma, Italy
}

Received 18 March 2019 / Accepted 4 June 2019

\section{ABSTRACT}

\begin{abstract}
We present the results of an XMM-Newton observation of the slowly rotating $(P=3.4 \mathrm{~s})$, highly magnetized $\left(B \approx 3 \times 10^{13} \mathrm{G}\right)$ radio pulsar PSR J0726-2612. A previous X-ray observation with the Chandra satellite showed that some of the properties of PSR J0726-2612 are similar to those of the X-ray-dim isolated neutron stars (XDINSs), a small class of nearby slow pulsars characterized by purely thermal X-ray spectra and undetected in the radio band. We confirm the thermal nature of the X-ray emission of PSR J0726-2612, which can be fitted by the sum of two blackbodies with temperatures $k T_{1}=0.074_{-0.011}^{+0.006} \mathrm{keV}$ and $k T_{2}=0.14_{-0.02}^{+0.04} \mathrm{keV}$ and emitting radii $R_{1}=10.4_{-2.8}^{+10.8} \mathrm{~km}$ and $R_{2}=0.5_{-0.3}^{+0.9} \mathrm{~km}$, respectively (assuming a distance of $1 \mathrm{kpc}$ ). A broad absorption line modeled with a Gaussian profile centered at $0.39_{-0.03}^{+0.02} \mathrm{keV}$ is required in the fit. The pulse profile of PSR J0726-2612 is characterized by two peaks with similar intensity separated by two unequal minima, a shape and pulsed fraction that cannot be reproduced without invoking magnetic beaming of the X-ray emission. The presence of a single radio pulse suggests that in PSR J0726-2612 the angles that the dipole axis and the line of sight make with the rotation axis, $\xi$ and $\chi$, respectively, are similar. This geometry differs from that of the two radio-silent XDINSs with double-peaked pulse profiles similar to that of PSR J0726-2612, for which $\xi \sim 90^{\circ}$ and $\chi \sim 45^{\circ}$ have recently been estimated. These results strengthen the similarity between PSR J0726-2612 and the XDINSs and support the possibility that the lack of radio emission from the latter might simply be due to an unfavorable viewing geometry.
\end{abstract}

Key words. pulsars: general - pulsars: individual: PSR J0726-2612 - stars: neutron - X-rays: stars

\section{Introduction}

Observations with the ROSAT satellite in the mid-1990s led to the discovery of a small group of isolated neutron stars characterized by thermal emission at soft X-rays, now known as XDINSs (X-ray-dim isolated neutron stars; see Haberl 2007; Turolla 2009 for reviews). The XDINSs have spin periods in the range $P \sim$ $3-17 \mathrm{~s}$ and period derivatives of a few $10^{-14} \mathrm{~s} \mathrm{~s}^{-1}$, which result in characteristic ages of $\tau_{\mathrm{c}}=P / 2 \dot{P} \sim 1-4 \mathrm{Myr}$. With the usual assumption that the spin-down is due to magnetic dipole braking, these timing parameters imply magnetic fields of the order of a few $10^{13} \mathrm{G}$.

The XDINSs are at distances of only a few hundred parsecs and for two of them the parallax of the optical counterpart has been measured (Walter et al. 2010; Tetzlaff et al. 2011). The XDINSs have X-ray luminosities of $10^{31}-10^{32} \mathrm{erg} \mathrm{s}^{-1}$, higher than their spin-down power. Their X-ray spectra are very soft, with blackbody temperatures of $k T \sim 45-110 \mathrm{eV}$, often showing the presence of broad absorption lines. If these lines are interpreted as proton cyclotron features or atomic transitions (see, e.g., Kaplan et al. 2008), the magnetic fields estimated from their energies are of the same order as those derived from the spin-down rate assuming magnetic dipole braking. The X-ray emission of XDINSs, con- sisting only of thermal components, is believed to come directly from the star surface and, given the small distance of these sources, it is little affected by photoelectric absorption in the interstellar medium. The discovery of XDINS caused some excitement since they appeared as optimal targets to test neutron star surface emission models without being affected by the presence of nonthermal emission. However, the ultimate goal of constraining the star radius and hence the equation of state with these studies is still hampered by our poor knowledge of the composition and magnetization of the surface layers of neutron stars.

The attempt to explain the different manifestations of neutron stars (e.g., Mereghetti 2011) in the context of a unified evolutionary picture is one of the current challenges in the study of neutron stars (Kaspi 2010; Igoshev et al. 2014). In the $P-\dot{P}$ diagram, shown in Fig. 1, XDINSs are located in the region below that occupied by the magnetars, a group of isolated neutron stars powered mainly by magnetic energy (see, e.g. Mereghetti et al. 2015; Turolla et al. 2015; Kaspi \& Beloborodov 2017). This has led to the suggestion that the XDINSs might be the descendants of magnetars (Heyl \& Kulkarni 1998; Colpi et al. 2000). The strong internal field of magnetars $\left(B \gtrsim 10^{15} \mathrm{G}\right)$ significantly affects their thermal evolution (Viganò et al. 2013), resulting in luminosities higher than those predicted for normal pulsars of a similar age. 


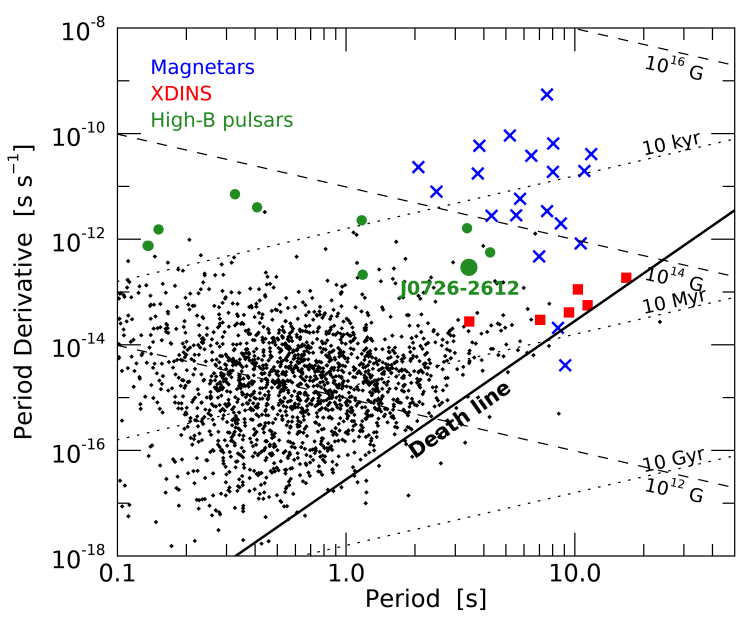

Fig. 1. $P-\dot{P}$ diagram of rotation-powered pulsars (black dots) and other classes of isolated pulsars (colored symbols). Lines of equal characteristic age (dotted, $10^{4}-10^{10} \mathrm{yr}$ ) and equal dipole magnetic field (dashed, $\left.10^{12}-10^{16} \mathrm{G}\right)$ are indicated. The radio pulsar death line $B / P^{2}=1.7 \times$ $10^{11} \mathrm{G} \mathrm{s}^{-2}$ (Bhattacharya et al. 1992) is also shown. The data are taken from the ATNF Pulsar Catalogue (Manchester et al. 2005).

A distinctive property of the XDINSs is that they are not detected in the radio band ${ }^{1}$ (Kondratiev et al. 2009). The reason for the lack of radio emission is still uncertain. One possibility is that this is due to their old age and long spin period (Baring \& Harding 1998, 2001). However, a few radio pulsars with periods $\gtrsim 10 \mathrm{~s}$ have recently been discovered: PSR J0250+5854 with $P=23.5$ s (Tan et al. 2018), and a second one with $P=12.1 \mathrm{~s}$ (Morello et al., in prep). Another explanation might be related to the geometrical configuration of their magnetosphere, that, especially if they are old magnetars, might be strongly nondipolar (Turolla et al. 2015). Finally, it cannot be excluded that (at least some of) the XDINSs are simply ordinary radio pulsars with radio beams unfavorably aligned with respect to the Earth. In this respect, it is interesting to investigate radio-loud pulsars with X-ray properties and/or timing parameters similar to those of the XDINSs, such as the long-period (greater than a few seconds) and high- $B\left(>10^{13} \mathrm{G}\right)$ pulsars.

Among these, here we focus on PSR J0726-2612, a radio pulsar with spin period $P=3.44 \mathrm{~s}$ and characteristic age of $200 \mathrm{kyr}$ that was discovered in the Parkes High-Latitude Survey (Burgay et al. 2006). Its timing parameters (Table 1) are in the range of those of the XDINSs. The similarity with the XDINSs was reinforced by X-ray observations with the Chandra satellite (Speagle et al. 2011), that revealed a soft thermal spectrum with blackbody temperature $k T \approx 87 \mathrm{eV}$, and pulsations with a sinusoidal, double-peaked profile. The distance of PSR J0726-2612 is unknown. Its dispersion measure DM $=69.4 \pm 0.4 \mathrm{~cm}^{-3} \mathrm{pc}$ (Burgay et al. 2006) implies a distance $d=2.9 \mathrm{kpc}$, assuming the Galactic electrons distribution of Yao et al. (2017). However, there are a few facts suggesting that this is probably an overestimate. For example, such a large value for $d$ would give a distance of $230 \mathrm{pc}$ from the Galactic plane, implying that if PSR J0726-2612 was born close to the plane and its true age were similar to $\tau_{\mathrm{c}}$, its velocity would be of the order of a thousand kilometers per second. This value is not impossible, but it would be at the far end of the pulsar velocity distribution (Hobbs et al. 2005). More importantly, for such a large $d$, one would expect an X-ray absorption corresponding to a size-

\footnotetext{
1 The possible detection of pulsed emission from two XDINSs at very
} low frequencies (Malofeev et al. 2005, 2006) is so far unconfirmed.
Table 1. Observed and derived parameters for PSR J0726-2612.

\begin{tabular}{|c|c|}
\hline RA (J2000.0). & $07^{\mathrm{h}} 26^{\mathrm{m}} 08^{\mathrm{s}} \cdot 12(4)$ \\
\hline $\operatorname{Dec}(\mathrm{J} 2000.0)$. & $-26^{\circ} 12^{\prime} 38^{\prime \prime} .1(8)$ \\
\hline Period $P(\mathrm{~s}) \ldots$ & $3.4423084877(4)$ \\
\hline Period derivative $\dot{P}\left(\mathrm{~s} \mathrm{~s}^{-1}\right)$ & $2.9311(4) \times 10^{-13}$ \\
\hline Epoch (MJD) .......... & 52950 \\
\hline Characteristic age $\tau_{\mathrm{c}}$ (years) $\ldots \ldots \ldots \ldots$ & $1.86 \times 10^{5}$ \\
\hline Surface dipolar magnetic field $B_{\mathrm{s}}(\mathrm{G}) \ldots \ldots$ & $3.2 \times 10^{13}$ \\
\hline Rotational energy loss rate $\dot{E}\left(\mathrm{erg} \mathrm{s}^{-1}\right) \ldots$ & $2.8 \times 10^{32}$ \\
\hline Dispersion measure DM $\left(\mathrm{cm}^{-3} \mathrm{pc}\right) \ldots \ldots$ & $69.4(4)$ \\
\hline
\end{tabular}

Notes. Data are taken from Burgay et al. (2006) and the ATNF Pulsar Catalogue (Manchester et al. 2005). Numbers in parentheses show the $1 \sigma$ uncertainty for the last digits.

able fraction of the total Galactic H I column density, which in this direction is $\sim 5 \times 10^{21} \mathrm{~cm}^{-1}$ (Kalberla et al. 2005), while the observed value is a factor ten smaller. Finally, the line of sight toward PSR J0726-2612 crosses the Gould belt, which is not included in the electron distribution model of Yao et al. (2017). This could explain the large distance inferred from the DM. This local structure $(d \sim 200-400 \mathrm{pc})$ comprises several OB associations that have been proposed as the birthplace of the XDINSs (Popov et al. 2003, 2005). Speagle et al. (2011) suggested that PSR J0726-2612 could also be associated with the Gould belt and hence be closer than $\sim 1 \mathrm{kpc}$.

Here we report the results of XMM-Newton observations which show other similarities between PSR J0726-2612 and the XDINSs. In the following we scale all the distance-dependent quantities to $d_{\mathrm{kpc}}=1 \mathrm{kpc}$ and adopt representative values of mass and radius of $1.2 M_{\odot}$ and $12 \mathrm{~km}$, respectively.

\section{Observations and data reduction}

PSR J0726-2612 was observed with the European Photon Imaging Cameras (EPIC) instrument on board XMM-Newton with a single pointing lasting $108 \mathrm{ks}$ on 2013 April 8. The three cameras of EPIC (0.1-12 keV), the pn (Strüder et al. 2001), and the two MOS (Turner 2001), were operated in Full Frame mode with the thin optical filter. While the EPIC-pn time resolution $(73.4 \mathrm{~ms})$ is adequate to reveal the pulsations of the source, this is impossible for the EPIC-MOS given its resolution time of $2.6 \mathrm{~s}$.

The data reduction was performed using the EPPROC and EMPROC pipelines of version 15 of the Science Analysis System $(\mathrm{SAS})^{2}$. We selected single- and multiple-pixel events $($ PATTERN $\leq 4$ and PATTERN $\leq 12$ ) for both the EPIC-pn and -MOS. We then removed time intervals of high background using the SAS program ESPFILT with standard parameters. The source was detected by EPIC at coordinates RA $=07^{\mathrm{h}} 26^{\mathrm{m}} 08.1$, Dec $=-26^{\circ} 12^{\prime} 38^{\prime \prime}$, fully consistent with the radio position (Table 1). The source events were selected from a circle of radius $40^{\prime \prime}$ centered at the radio position, while the background was extracted from a nearby circular region of radius $60^{\prime \prime}$. The resulting net exposure times and source events are listed in Table 2. At the corresponding count rates pile-up effects are not relevant.

\section{Results}

\subsection{Timing analysis}

PSR J0726-2612 is barely detected above $1.5 \mathrm{keV}$, therefore we limited our timing analysis to the energy band $0.15-1.5 \mathrm{keV}$.

\footnotetext{
2 https://wWW. cosmos. esa.int/web/xmm-newton/sas
} 
Table 2. Exposure times and source counts for PSR J0726-2612 in the three EPIC cameras.

\begin{tabular}{lccc}
\hline \hline Data & EPIC camera & $\begin{array}{c}\text { Exposure time } \\
\text { ks }\end{array}$ & $\begin{array}{c}\text { Source counts } \\
0.15-1.5 \mathrm{keV}\end{array}$ \\
\hline Phase-averaged & pn & 37.8 & $18938 \pm 140$ \\
& MOS1 & 64.0 & $4499 \pm 69$ \\
& MOS2 & 70.4 & $5212 \pm 74$ \\
\hline Min 1 & pn & 9.4 & $3823 \pm 63$ \\
Max 1 & pn & 9.4 & $5576 \pm 76$ \\
Min 2 & pn & 9.4 & $4088 \pm 65$ \\
Max 2 & pn & 9.4 & $5447 \pm 75$ \\
\hline
\end{tabular}

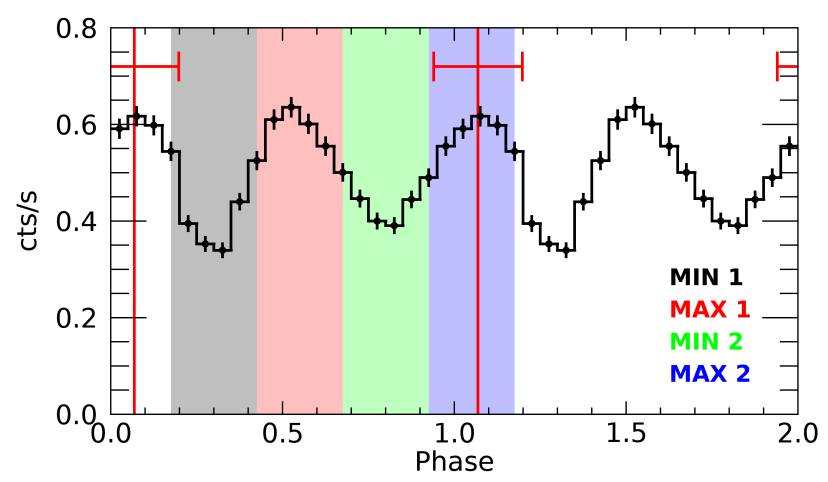

Fig. 2. Pulse profile of PSR J0726-2612 in the energy range 0.15$1.5 \mathrm{keV}$ obtained by folding the EPIC-pn data in 20 phase bins at the period derived from the radio ephemeris (Table 1). The vertical red line represents the location of the radio pulse (derived from Speagle et al. 2011), with its uncertainty $(1 \sigma)$. The colored bands indicate the intervals used for the phase-resolved spectroscopy.

The times of arrival were converted to the barycenter of the solar system with the task BARYCEN. An epoch folding search of the EPIC-pn data gave a best period of $P=3.442396(1) \mathrm{s}$, which is consistent within $0.7 \sigma$ with the value expected at the XMM-Newton observation epoch (56390 MJD) using the ATNF ephemeris reported in Table 1. The background-subtracted light curve in the energy band $0.15-1.5 \mathrm{keV}$ is shown in Fig. 2. The position of the radio pulse is indicated, with its $1 \sigma$ uncertainty, as a vertical red line.

The EPIC-pn pulse profile shows two peaks with the same intensity (net count rate of $\max _{1}=0.62 \pm 0.02 \mathrm{cts} \mathrm{s}^{-1}$ and $\max _{2}=0.64 \pm 0.02$ cts s$\left.^{-1}\right)$, separated by about 0.5 cycles. The two minima of the pulse profile are instead significantly different: $\min _{1}=0.34 \pm 0.01 \mathrm{cts} \mathrm{s}^{-1}$ and $\min _{2}=0.39 \pm 0.01 \mathrm{cts} \mathrm{s}^{-1}$. The pulse profile is symmetric in phase with respect to any of the two minima, but a fit with a constant plus a sine function at half of the spin period is not acceptable $\left(\chi_{v}^{2}=2.7\right.$ for 17 d.o.f. $)$. The pulsed fraction ${ }^{3}$ is $30 \pm 2 \%$.

Figure 3 shows that the soft $(0.15-0.4 \mathrm{keV})$ and hard energy ranges $(0.4-1.5 \mathrm{keV})$ have slightly different pulsed fractions: $26 \pm 3 \%$ and $37 \pm 3 \%$, respectively. Moreover, the positions of the first minimum and of the second maximum are shifted of about 1 bin between the two energy ranges, but the symmetry around the minima is preserved in both bands. Fits with a constant plus sine function give $\chi_{v}^{2}=1.6$ and $\chi_{v}^{2}=3.5$ for the soft and hard profiles, respectively. The hardness ratio ${ }^{4}$, shown in the

\footnotetext{
3 Defined as $(\max (\mathrm{CR})-\min (\mathrm{CR})) /(\max (\mathrm{CR})+\min (\mathrm{CR}))$, where $\mathrm{CR}$ is the background-subtracted count rate.

4 Defined as (hard(CR) $-\operatorname{soft}(\mathrm{CR})) /($ hard(CR) + soft(CR)), where the soft energy range is $0.15-0.4 \mathrm{keV}$, the hard one $0.4-1.5 \mathrm{keV}$.
}

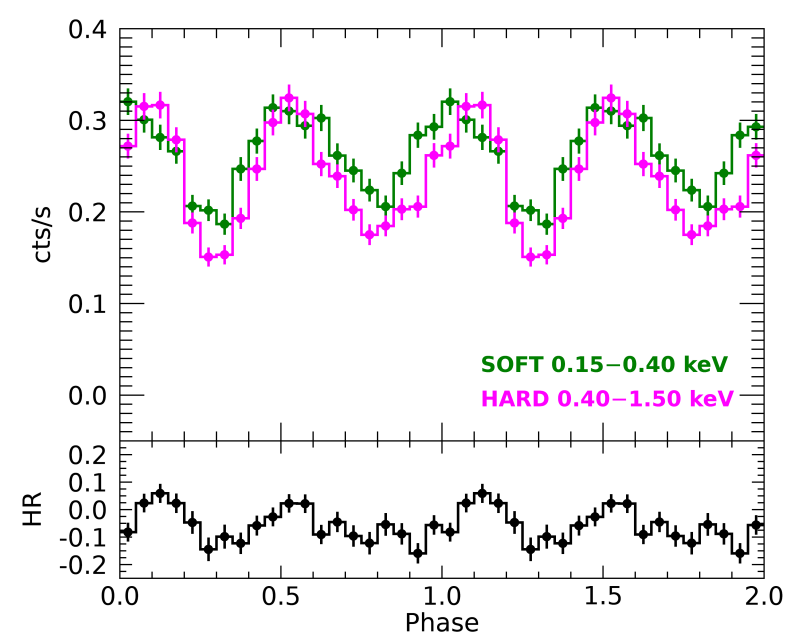

Fig. 3. EPIC-pn light curve of PSR J0726-2612 (20 phase bins) in the energy ranges $0.15-0.4-1.5 \mathrm{keV}$ together with the corresponding hardness ratio.

lower panel of the same figure clearly indicates the presence of phase-dependent spectral variations: the source is softer during the minima and harder during the maxima.

\subsection{Spectral analysis}

The spectral analysis was performed using XSPEC (version 12.8.2). The spectra were rebinned using the GRPPHA tool with a minimum of 50 counts per bin. The spectra of the three cameras were fitted simultaneously, including a renormalization factor to account for possible cross-calibration uncertainties. Errors on the spectral parameters are at $1 \sigma$ confidence level.

We used the photoelectric absorption model TBABs, with cross sections and abundances from Wilms et al. (2000). Both a single power law and a blackbody did not provide acceptable fits, giving $\chi_{v}^{2} \approx 6$ and $\chi_{v}^{2}=1.37$ for 213 d.o.f. (null-hypothesis probability, nhp, of $3 \times 10^{-4}$ ), respectively. We then attempted a fit with magnetized hydrogen atmosphere models (NSA and NSMAXG in XSPEC, Pavlov et al. 1995; Ho et al. 2008, 2014). However, neither of the two sets of available models (the first with a single surface $B$ and $T_{\text {eff }}$, the second with $B$ and $T_{\text {eff }}$ varying across the surface according to the magnetic dipole model) gave an acceptable fit $\left(\chi_{v}^{2}>2.2\right.$ for 213 d.o.f.). In conclusion, we could not find a good fit with single-component models.

Furthermore, modeling the spectra with a blackbody plus power law or with the sum of two blackbodies was unsatisfactory. With the former we obtained a negative photon index for the power law, while with the latter, the second thermal component had a negligible flux, and did not improve the quality of the fit with respect to that of a single blackbody $\left(\chi_{v}^{2}=1.32\right.$ for 211 d.o.f., $\operatorname{nhp}=10^{-3}$ ).

A real improvement in the fit was obtained by adding to the blackbody a broad absorption line modeled with a Gaussian (GBB) centered at $E=1.09 \pm 0.09 \mathrm{keV}$ and width $\sigma=$ $0.28 \pm 0.08 \mathrm{keV}\left(\chi_{v}^{2}=1.12\right.$ for 210 d.o.f.). Following the recent results of Yoneyama et al. (2019), we explored the possibility of adopting a two-blackbody component model plus a Gaussian line in absorption (G2BB). With this model we found a good fit with the line placed at $E=0.39_{-0.03}^{+0.02} \mathrm{keV}$ and with a broadening of $\sigma=0.08_{-0.02}^{+0.03} \mathrm{keV}\left(\chi_{v}^{2}=1.00\right.$ for 208 d.o.f.). The addition of the line yields an improvement of the $\chi^{2}$ of $F=\chi_{2 \mathrm{BB}}^{2} / \chi_{\mathrm{G} 2 \mathrm{BB}}^{2}=1.32$. To assess the statistical significance of the line, we estimated 


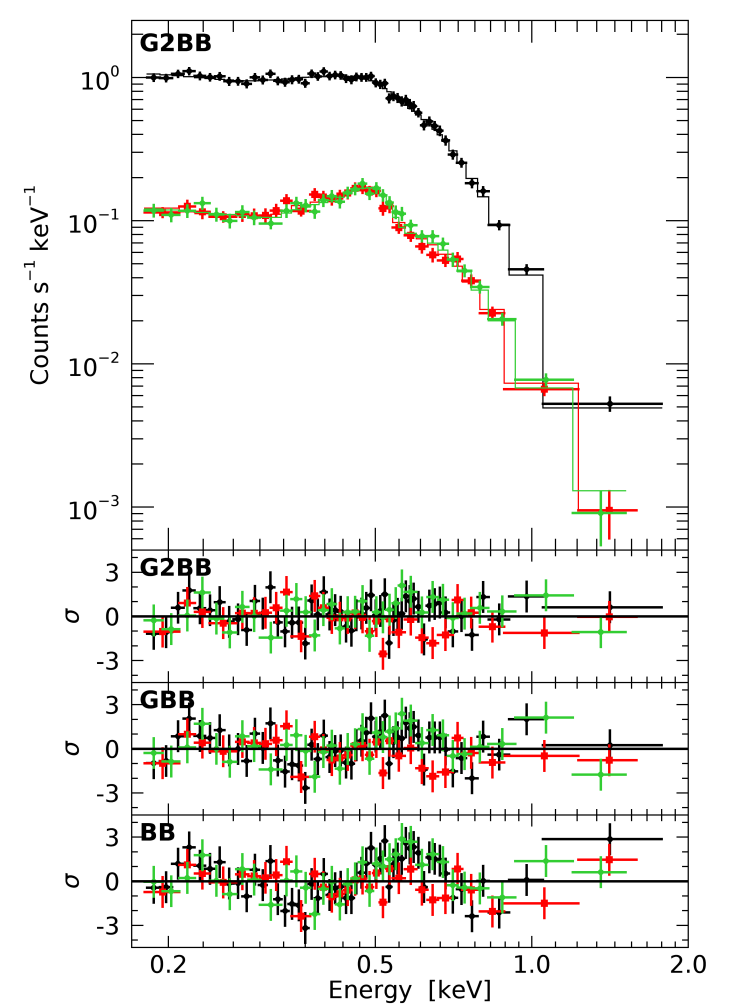

Fig. 4. EPIC-pn (black), -MOS1 (red) and -MOS2 (green) phaseaveraged spectra of PSR J0726-2612. Top panel: best fit using a Gaussian absorption feature at $E=0.39 \mathrm{keV}$ and two blackbodies (G2BB). Lower panels: residuals of the best fit (G2BB), of a Gaussian absorption feature at $E=1.09 \mathrm{keV}$ and one blackbody (GBB), and of a single blackbody (BB) in units of $\sigma$. Data have been rebinned for display purposes only.

through Monte Carlo simulations the probability of obtaining by chance an equal (or better) fit improvement: we estimate a probability of $\sim 10^{-5}$ of having $F \geq 1.32$, corresponding to a $\sim 4.4 \sigma$ significance of the line. The cold blackbody $\left(k T_{1} \approx 0.074 \mathrm{keV}\right)$ has an emitting radius $R_{1}=10.4_{-2.8}^{+10.8} d_{\mathrm{kpc}} \mathrm{km}$, compatible with emission from the whole neutron star, while the hot blackbody has $k T_{2} \approx 0.14 \mathrm{keV}$ and $R_{2}=0.5_{-0.3}^{+0.9} d_{\mathrm{kpc}} \mathrm{km}$.

A good fit was also found with the magnetized atmosphere models with a dipole distribution of the surface magnetic field ( $B=10^{13} \mathrm{G}$ at the poles) plus a Gaussian line in absorption. With the NSA model, we found an effective temperature $T_{\text {eff }}=0.40 \pm 0.08 \mathrm{MK}$ (corresponding to an observed temperature $k T=0.029 \pm 0.001 \mathrm{keV}), d=121_{-12}^{+13} \mathrm{pc}$, and $E=$ $0.37_{-0.03}^{+0.02} \mathrm{keV}, \sigma=0.09_{-0.01}^{+0.02} \mathrm{keV}$ for the Gaussian line $\left(\chi_{v}^{2}=1.03\right.$ for 210 d.o.f.). With the NSMAXG model, for an impact parameter (that is the angle between the line of sight and the dipole axis) $\eta=90^{\circ}$, the model parameters are $T_{\text {eff }}=0.39 \pm 0.02 \mathrm{MK}$ $(k T=0.028 \pm 0.001 \mathrm{keV}), d=63_{-17}^{+26} \mathrm{pc}$ and $E=0.28 \pm 0.09$ $\mathrm{keV}, \sigma=0.14_{-0.04}^{+0.06} \mathrm{keV}$ for the Gaussian line $\left(\chi_{v}^{2}=1.02\right.$ for 210 d.o.f.). Using instead the same model with $\eta=0^{\circ}$, the fit was not acceptable $\left(\chi_{v}^{2}=2.38\right.$ for 210 d.o.f. $)$.

The spectral results are summarized in Table 3 , while in Fig. 4 the best blackbody fits are shown.

The light curves and hardness ratio shown in Fig. 3 indicate that a spectral variation occurs as a function of the rotation phase. Therefore, we extracted the EPIC-pn spectra of the phase intervals corresponding to the two minima and the two maxima of the pulse profile, as shown in Fig. 2 (the number of source events in each spectrum is listed in Table 2). In order to illustrate the spectral

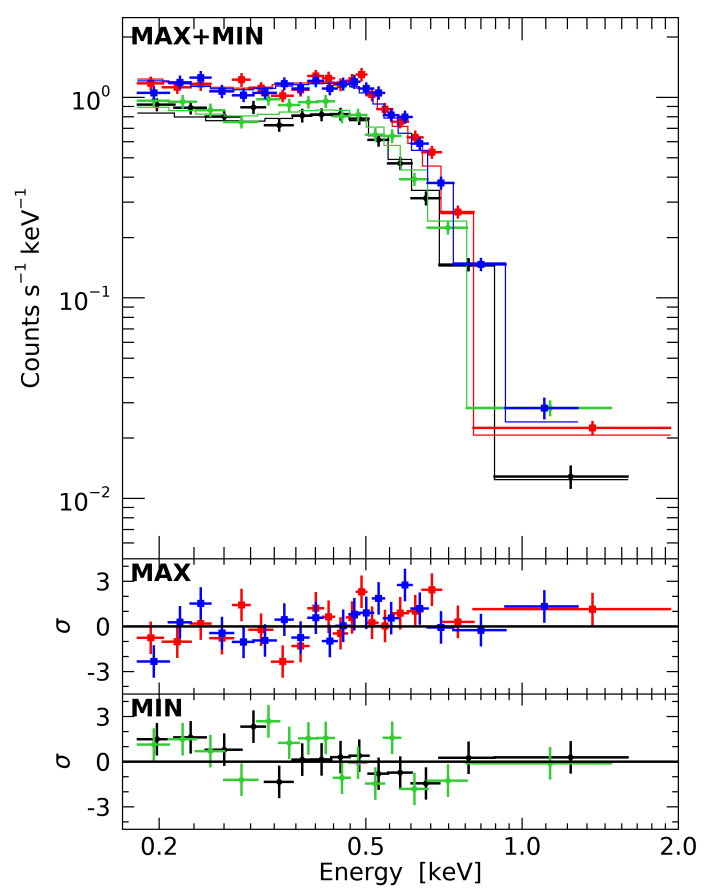

Fig. 5. EPIC-pn phase-resolved spectra fitted with the G2BB model used for the phase-averaged spectra (the color code is the same as in Fig. 2). The overall normalization is the only free parameter. The residuals of the spectra at maxima and minima, in units of $\sigma$, are shown in the lower panels. Data have been rebinned for display purposes only.

variations, we fitted the spectra with the G2BB model, fixing all of the parameters at the best fit values of the phase-averaged spectrum, except for an overall normalization. The residuals shown in the two lower panels of Fig. 5 indicate that the spectra of the two maxima are similar and significantly harder than those of the minima. Their normalization factors with respect to the phaseaveraged spectrum are consistent $\left(N_{\max 1}=1.16 \pm 0.02\right.$ and $\left.N_{\max 2}=1.14 \pm 0.02\right)$, while those of the two minima are different $\left(N_{\min 1}=0.85 \pm 0.01\right.$ and $\left.N_{\min 2}=0.80 \pm 0.01\right)$.

We then fitted the four spectra separately, keeping only the interstellar absorption and the parameters of the cold blackbody fixed, because we do not expect them to vary during a stellar rotation. The results are given in Table 3 . The absorption line is at the same energy in the four spectra, but it has different widths and normalizations. The hot blackbody temperature is lower $(k T \approx$ $0.11 \mathrm{keV})$ and its emission radius is $\operatorname{larger}\left(R \approx 1.5 d_{\mathrm{kpc}} \mathrm{km}\right)$ at the two maximum phases than at the first minimum $(k T \approx 0.17 \mathrm{keV}$ and $R \approx 0.20 d_{\mathrm{kpc}} \mathrm{km}$ ), while these parameters are poorly constrained at the second minimum. We also tried other fits allowing more parameters to vary, but the results were inconclusive due to the strong parameter degeneracy.

\section{Discussion}

Our XMM-Newton results for PSR J0726-2612 are consistent with those previously obtained with Chandra (Speagle et al. 2011), but thanks to a significant detection with good statistics over a broader energy range, they provide more information on the spectrum and pulse profile of this pulsar.

\subsection{The X-ray spectrum}

We found that the spectrum of PSR J0726-2612 is more complex than the single blackbody that was adequate to fit the 
Table 3. Results for the phase-averaged and phase-resolved spectra of PSR J0726-2612.

\begin{tabular}{|c|c|c|c|c|c|c|c|c|c|c|c|}
\hline Model & $\begin{array}{c}N_{\mathrm{H}}{ }^{(a)} \\
10^{20} \mathrm{~cm}^{-2}\end{array}$ & $\begin{array}{l}k T_{1} \\
\mathrm{keV}\end{array}$ & $\begin{array}{l}R_{1}{ }^{(b)} \\
\mathrm{km}\end{array}$ & $\begin{array}{l}k T_{2} \\
\mathrm{keV}\end{array}$ & $\begin{array}{l}R_{2}{ }^{(b)} \\
\mathrm{km}\end{array}$ & $\begin{array}{c}E \\
\mathrm{keV}\end{array}$ & $\begin{array}{c}\sigma \\
\mathrm{keV}\end{array}$ & $\begin{array}{c}\text { Strength }{ }^{(c)} \\
\mathrm{keV}\end{array}$ & $\begin{array}{c}F_{\text {unabs }}^{0.1-2} \\
\mathrm{erg} \mathrm{s}^{-1} \mathrm{~cm}^{-2}\end{array}$ & $\chi_{v}^{2} /$ d.o.f. & nhp \\
\hline \multicolumn{12}{|c|}{ Phase-averaged spectra: } \\
\hline BB & $4.1 \pm 0.2$ & $0.0896(6)$ & $4.90 \pm 0.15$ & $\ldots$ & $\ldots$ & $\ldots$ & $\ldots$ & $\ldots$ & $1.60_{-0.05}^{+0.06}$ & $1.37 / 213$ & $3 \times 10^{-4}$ \\
\hline $2 \mathrm{BB}$ & $4.3 \pm 0.2$ & $0.0888(7)$ & $5.1 \pm 0.2$ & $>0.33$ & $<0.018$ & $\ldots$ & $\ldots$ & $\ldots$ & $1.65_{-0.06}^{+0.07}$ & $1.32 / 211$ & $1 \times 10^{-3}$ \\
\hline GBB & $2.8 \pm 0.3$ & $0.11 \pm 0.01$ & $2.9_{-0.4}^{+0.5}$ & $\ldots$ & $\ldots$ & $1.09 \pm 0.09$ & $0.28 \pm 0.08$ & $1.0_{-0.6}^{+1.1}$ & $1.37_{-0.09}^{+0.30}$ & $1.12 / 210$ & 0.11 \\
\hline $\mathrm{G} 2 \mathrm{BB}$ & $5.3_{-0.8}^{+1.2}$ & $0.074_{-0.011}^{+0.006}$ & $10.4_{-2.8}^{+0.4}$ & $0.14_{-0.02}^{+0.04}$ & $0.5_{-0.3}^{+0.9}$ & $0.39_{-0.03}^{+0.02}$ & $0.08_{-0.02}^{+0.03}$ & $0.12_{-0.05}^{+0.13}$ & $3.30_{-0.85}^{+3.85}$ & $1.00 / 208$ & 0.47 \\
\hline $\operatorname{GNSA}^{(e)}$ & $6.9_{-11}^{+0.8}$ & $0.029(1)$ & $14.3^{-2.8}$ & $\ldots$ & $\ldots$ & $0.37_{-0.03}^{-0.03}$ & $0.09_{-0.01}^{+0.02}$ & $0.17_{-0.04}^{-0.03}$ & $9.0 \pm 1.1$ & $1.03 / 210$ & 0.36 \\
\hline $\operatorname{GNSMAXG}^{(f)}$ & $5.9_{-4.2}^{-1.1}$ & $0.028(1)$ & $14.3^{(d)}$ & $\ldots$ & $\ldots$ & $0.28 \pm 0.09$ & $0.14_{-0.04}^{-0.01}$ & $0.62_{-0.33}^{-1.264}$ & $19.3_{-7.0}^{+9.7}$ & $1.02 / 210$ & 0.40 \\
\hline \multicolumn{12}{|c|}{ G2BB phase-resolved: } \\
\hline Maxima 1 & $5.3^{(d)}$ & $0.074^{(d)}$ & $10.4^{(d)}$ & $0.110(7)$ & $1.55_{-0.30}^{+0.40}$ & $0.39 \pm 0.01$ & $0.07 \pm 0.01$ & $0.11_{-0.01}^{+0.02}$ & $3.6 \pm 0.2$ & $1.00 / 80$ & 0.47 \\
\hline Maxima 2 & $5.3^{(d)}$ & $0.074^{(d)}$ & $10.4^{(d)}$ & $0.111(9)$ & $1.40_{-0.35}^{+0.45}$ & $0.39 \pm 0.01$ & $0.06 \pm 0.01$ & $0.10 \pm 0.01$ & $3.6 \pm 0.2$ & $0.95 / 78$ & 0.61 \\
\hline Minima 1 & $5.3^{(d)}$ & $0.074^{(d)}$ & $10.4^{(d)}$ & $0.17_{-0.04}^{+0.07}$ & $0.20_{-0.13}^{+0.31}$ & $0.40_{-0.02}^{+0.01}$ & $0.14_{-0.02}^{+0.04}$ & $0.23_{-0.03}^{+0.05}$ & $2.90_{-0.25}^{+0.45}$ & $1.14 / 49$ & 0.23 \\
\hline Minima 2 & $5.3^{(d)}$ & $0.074^{(d)}$ & $10.4^{(d)}$ & $0.29_{-0.09}^{+0.74}$ & $0.06_{-0.03}^{+0.05}$ & $0.39_{-0.03}^{+0.02}$ & $0.13_{-0.02}^{+0.03}$ & $0.20_{-0.02}^{+0.04}$ & $3.1_{-0.2}^{+0.3}$ & $1.28 / 52$ & 0.08 \\
\hline
\end{tabular}

Notes. Joint fits of EPIC-pn+MOS1+MOS2 phase-averaged spectra and EPIC-pn phase-resolved spectra of PSR J0726-2612. The fluxes, corrected for the absorption, are expressed in units of $10^{-12} \mathrm{erg} \mathrm{cm}^{-2} \mathrm{~s}^{-1}$. Temperatures and radii are observed quantities at infinity. Errors at $1 \sigma$. ${ }^{(a)}$ Derived with the photoelectric absorption model TBABs (Wilms et al. 2000). ${ }^{(b)}$ Radius for an assumed distance of 1 kpc. ${ }^{(c)}$ Parameter of GABS model such as the optical depth at line center is $\tau=\operatorname{strength} / \sqrt{2 \pi} \sigma .{ }^{(d)}$ Fixed value. ${ }^{(e)}$ NSA model (Pavlov et al. 1995) with $M=1.2 M_{\odot}, R=12 \mathrm{~km}$, $B=10^{13} \mathrm{G}$ and a uniform temperature distribution. This model yields a best-fit distance $d=121_{-12}^{+13}$ pc. ${ }^{(f)}$ NSMAXG model (Ho et al. 2008, 2014) with $M=1.2 M_{\odot}, R=12 \mathrm{~km}$, a dipole distribution of the magnetic field ( $B=10^{13} \mathrm{G}$ at the poles) and consistent temperature distribution, seen with $\eta=90^{\circ}$. This model yields a best-fit distance of $d=63_{-17}^{+26} \mathrm{pc}$.

Chandra data. The single blackbody fit requires the addition of a broad absorption line at $E \approx 1.09 \mathrm{keV}$. A better fit was obtained with two blackbody components, but also in this case a line at $E \approx 0.39 \mathrm{keV}$ is required. The colder blackbody component has an emitting area consistent with a large fraction of the star surface $\left(R_{1}=10.4_{-2.8}^{+10.8} d_{\mathrm{kpc}} \mathrm{km}\right)$, while the hotter one can be attributed to a small hot spot $\left(R_{2}=0.5_{-0.3}^{+0.9} d_{\mathrm{kpc}} \mathrm{km}\right)$, likely located at the magnetic pole.

Our results confirm that the interstellar absorption is about a factor of ten smaller than the value $\left(N_{\mathrm{H}}=2.1 \times 10^{21} \mathrm{~cm}^{-2}\right)$ inferred from the dispersion measure and the usual assumption of a $10 \%$ ionization of the interstellar medium (He et al. 2013). This might be due to the line of sight crossing the Gould belt.

An equally good fit was obtained with a magnetized hydrogen atmosphere covering the whole surface of the star, but also in this case the presence of an absorption line at $E \approx 0.37 \mathrm{keV}$ (NSA model) or $E \approx 0.28 \mathrm{keV}$ (NSMAXG model) is required. We note that the constant (polar) value of the magnetic field in the NSA (NSMAXG) model is fixed in the fits at $B=10^{13} \mathrm{G}$, and that the NSA model assumes a uniform distribution of the temperature. The NSMAXG model is more realistic, but it assumes that the dipole axis is orthogonal to the line of sight, which is not necessarily true for the case of PSR J0726-2612. Moreover, the inferred distance of $\approx 63$ pc seems unrealistically small.

The absorption lines we found in the spectra can be interpreted as proton cyclotron features at $E_{\text {cyc }}=0.063 B_{13} \times$ $(1+z) \mathrm{keV}$, where $z$ is the gravitational redshift and $B_{13}$ the magnetic field in units of $10^{13} \mathrm{G}$. In the case of G2BB model, for $E_{\text {cyc }}=0.39 \mathrm{keV}$ and $z \approx 0.2$, we get $B \approx 5 \times 10^{13} \mathrm{G}$, in good agreement with the dipole magnetic field evaluated at the poles $\left(B_{\mathrm{p}} \approx 6 \times 10^{13} \mathrm{G}\right)$. However, we caution that other explanations cannot be ruled out, including the possibility that the lines are simply an artefact resulting from an oversimplified modeling of the continuum emission. In fact, Viganò et al. (2014) showed that nonhomogeneus temperature distributions on a neutron star surface can in some cases lead to the appearance of broad features when the spectra are fitted with simple blackbody models.

\subsection{The $X$-ray pulse profile}

Contrary to the previous Chandra results, we also found that the double-peaked pulse profile of PSR J0726-2612 is not well described by a sinusoid, owing to the significant difference in the flux of the two minima. Remarkably, the pulse profile is symmetric for phase reflection around any of the two minima. Within the limits due to their lower statistics, these properties seem to hold also for the profiles in the soft and hard X-ray bands. The pulse profiles are moderately energy-dependent, with evidence for a harder emission in correspondence of the two peaks.

Although a detailed modeling of the light curves of PSR J0726-2612 is beyond the scope of the present work, we explored whether or not a simple model based on blackbody emission components with parameters consistent with the spectral results could reproduce the pulse profile. We assumed that the hotter blackbody comes from two antipodal magnetic polar caps with opening angle $\theta_{c, 1}=3^{\circ}$, while the colder one from two annuli extending between $\theta_{\mathrm{c}, 1}$ and $\theta_{\mathrm{c}, 2}=36^{\circ}$. The temperatures of the emitting regions were set to the values derived from the spectral analysis (model G2BB, $k T_{1}=0.074 \mathrm{keV}$, $k T_{2}=0.14 \mathrm{keV}$ ) and the angular apertures were chosen in such a way as to reproduce the emitting radii derived from the fit for a NS radius of $12 \mathrm{~km}$. We also added interstellar absorption and a Gaussian absorption line, with parameters fixed to those of the phase averaged spectrum. Synthetic light curves were computed using the method by Turolla \& Nobili (2013) and account for general-relativistic effects. We convolved the obtained light curves with the EPIC-pn instrumental response and evaluated the pulsed fraction in the energy range $0.15-1.5 \mathrm{keV}$. The results depend on the angles $\chi$ and $\xi$ that the rotation axis makes with the line of sight and the magnetic axis, respectively. As shown in Fig. 6, this simple model is unable to yield the observed pulsed fraction even for the most favorable geometry $(\mathrm{PF} \approx 21 \%$ for $\xi \approx \chi \gtrsim 35^{\circ}$ ). This is also true if only two antipodal point-like polar caps are considered, which is the configuration yielding the maximum pulsed fraction using isotropic emission (see e.g., Turolla \& Nobili 2013). Another problem is that, owing to the 


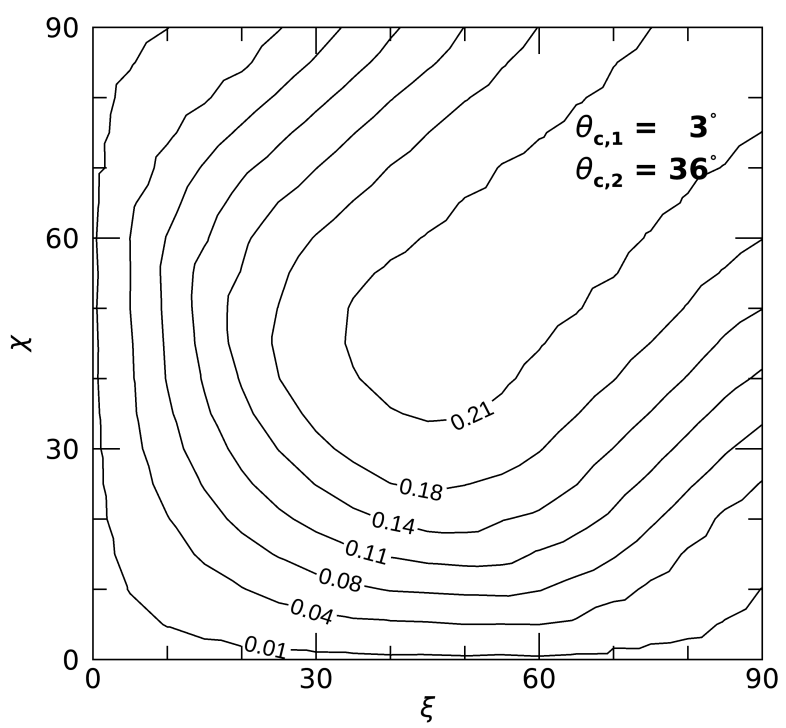

Fig. 6. Pulsed fraction for the G2BB model, where the blackbody emission comes from two, antipodal "cap+ring" spots centered on the magnetic poles. The aperture of the hot cap $(k T=0.14 \mathrm{keV})$ is $\theta_{\mathrm{c}, 1}=3^{\circ}$, while the colder $(k T=0.074 \mathrm{keV})$ ring extends from $\theta_{\mathrm{c}, 1}$ to $\theta_{\mathrm{c}, 2}=36^{\circ}$. The considered energy range is $0.15-1.5 \mathrm{keV}$, and a compactness of $M / R=0.1 M_{\odot} \mathrm{km}^{-1}$ has been assumed.

intrinsic symmetry of the model, the resulting light curves cannot exhibit different minima, as observed in PSR J0726-2612.

Indeed, this model is oversimplified and unlikely to apply to the real case. Whatever the mechanism responsible for the surface emission, the presence of a strong magnetic field results in some degree of anisotropy in the emitted radiation. In the case of a magnetized atmosphere, more complicated energy-dependent beaming patterns are produced; they consist of a relatively narrow pencil-beam aligned with the magnetic field, surrounded by a broader fan-beam at intermediate angles and account for most of the escaping radiation (see e.g., Pavlov et al. 1994). The angular pattern of the emerging intensity depends also on the local surface temperature and magnetic field, meaning that the morphology of the pulse profiles can be extremely variegated. Using a partially ionized hydrogen atmosphere model (Suleimanov et al. 2009) with improved opacities from Potekhin et al. (2014), we computed the expected pulse profiles, as described in Rigoselli et al. (2019). The best match with the data was obtained assuming emission from two antipodal hot spots with an effective temperature of $0.5 \mathrm{MK}$, and $\xi=30^{\circ}, \chi=35^{\circ}$. In Fig. 7 we show two examples with representative values of the magnetic field, $B=4 \times 10^{13} \mathrm{G}$ and $B=6 \times 10^{13} \mathrm{G}$. Although these pulse profiles qualitatively resemble that observed in PSR J0726-2612, we note that they have been computed considering only the X-ray emission from the polar caps. The addition of a contribution from an extended part of the star surface would reduce the pulsed fractions of the light curves shown in Fig. 7.

\subsection{Connections with the XDINSs}

Our spectral results, and in particular the presence of a broad absorption line, strengthen the similarity between PSR J0726-2612 and the XDINSs, for which similar spectral features have been reported (see Table 4). As illustrated in Fig. 8, not only the line properties, but also the best fit parameters of the continuum model are very similar to those recently reported in a systematic analysis of all the XDINS spectra with the G2BB model (Yoneyama et al. 2019).

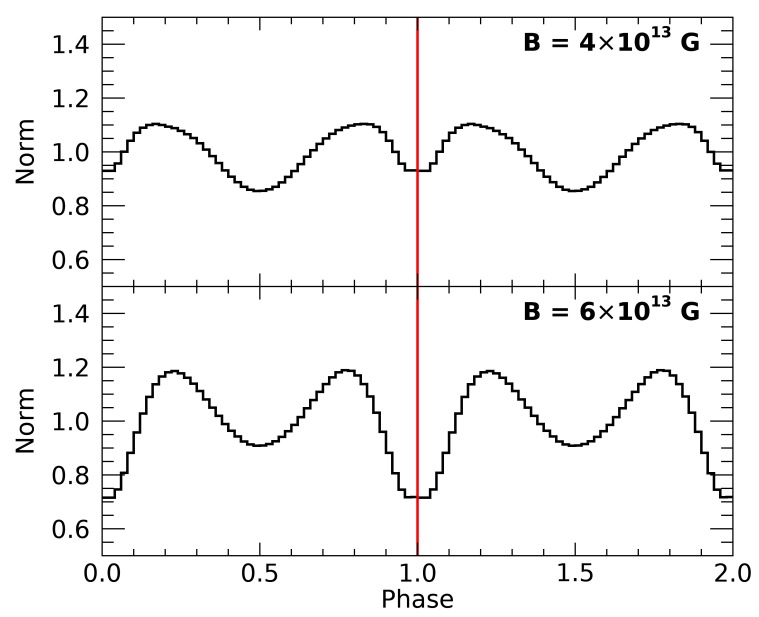

Fig. 7. Pulse profiles in the $0.15-1.5 \mathrm{keV}$ range in the case of emission from a hydrogen atmosphere model at two point-like polar caps with $T_{\text {eff }}=0.5 \mathrm{MK}$ and $B=4 \times 10^{13} \mathrm{G}$ (upper panel), and $B=6 \times 10^{13} \mathrm{G}$ (lower panel). We assumed $\xi=30^{\circ}, \chi=35^{\circ}$ and a compactness $M / R=$ $0.1 M_{\odot} \mathrm{km}^{-1}$. The vertical red line shows the phase expected for the radio peak.

The age-luminosity diagram shown in Fig. 9 gives even more strength to this analogy. The figure represents the bolometric luminosity of thermally emitting neutron stars as a function of their ages, characteristic or kinematic. The luminosity of PSR J0726-2612 $L_{\infty}=\left(4.0_{-1.0}^{+4.4}\right) \times 10^{32} \mathrm{erg} \mathrm{s}^{-1}$ corresponds to the cold component of the $\mathrm{G} 2 \mathrm{BB}$ fit to the phase-averaged spectrum (for $d=1 \mathrm{kpc}$ ). This component is in fact representative of the cooling emission from the entire star surface (the inclusion of the hot component would not significantly change the result, adding only about $3 \%$ to the total luminosity, well within the uncertainties). The observational data for other neutron stars are displayed in Fig. 9 as in Potekhin \& Chabrier (2018); most of them are taken from Viganò et al. (2013), with some updates and additions. The horizontal error bars show the uncertainties of kinematic ages, when available; otherwise the bars are replaced by arrows.

The position of PSR J0726-2612 in this diagram is indeed close to the group of XDINSs. Its place can be considered as intermediate between the regions occupied by ordinary neutron stars, which have either smaller luminosities or smaller ages, magnetars, which generally have larger luminosities, and XDINSs, which have somewhat smaller luminosities and larger ages. For comparison we plot two cooling curves, with heavy (nonaccreted) and light (accreted) chemical elements in the outer heat-blanketing envelope. The cooling curves are calculated for a neutron star of mass $M=1.2 M_{\odot}$ and the dipole magnetic field inferred for PSR J0726-2612 $\left(B_{p}=6 \times 10^{13} \mathrm{G}\right)$ using the code of Potekhin \& Chabrier (2018) with the equation of state BSk24 (Pearson et al. 2018), and singlet pairing-type superfluidity of neutrons and protons (according to Margueron et al. 2008 and Baldo \& Schulze 2007, respectively, both in the parametrized form of Ho et al. 2015). The triplet pairing-type superfluidity of neutrons is not included, because it is strongly suppressed by many-particle correlations, according to recent results of Ding et al. (2016). The latter suppression delays the onset of the Cooper pair breaking-formation mechanism of neutrino emission in the core of the neutron star and thus slows down the cooling, making the theoretical cooling curves compatible with the XDINS observations even without additional internal heating, which otherwise would be needed (e.g., Viganò et al. 2013).

While most of the XDINSs have single-peaked pulse profiles, two of them (RX J1308.6+2127, Hambaryan et al. 2011, and RX 
Table 4. Comparison between PSR J0726-2612 and the XDINSs.

\begin{tabular}{|c|c|c|c|c|c|c|c|c|c|}
\hline $\begin{array}{l}\text { Source } \\
\text { RX }\end{array}$ & $\begin{array}{l}P \\
\mathrm{~s}\end{array}$ & $\begin{array}{c}\dot{P} \\
10^{-14} \mathrm{~s} \mathrm{~s}^{-1}\end{array}$ & $\begin{array}{c}B_{\mathrm{p}} \\
10^{13} \mathrm{G}\end{array}$ & $\begin{array}{l}E_{\mathrm{cyc}} \\
\mathrm{eV}\end{array}$ & $\begin{array}{c}B_{\text {cyc }} \\
10^{13} \mathrm{G}\end{array}$ & $L_{X} / \dot{E}$ & Pulse & $\begin{array}{c}\mathrm{PF} \\
\%\end{array}$ & Refs. \\
\hline J0420.0-5022 & 3.45 & 2.76 & 2.0 & & & $0.31-0.38$ & Single & 13 & (1) \\
\hline J0720.4-3125 & 16.78 & 18.6 & 11.3 & $254_{-30}^{+25}$ & $3.4_{-0.4}^{+0.3}$ & $99-157$ & Double & 11 & (2) \\
\hline J0806.4-4123 & 11.37 & 5.6 & 5.1 & $241_{-12}^{+11}$ & $3.2_{-0.2}^{+0.2}$ & $10.6-16.7$ & Single & 6 & (1) \\
\hline J1308.6+2127 (RBS 1223) & 10.31 & 11.2 & 6.9 & $390_{-6}^{+6}$ & $5.16_{-0.08}^{+0.08}$ & $31.5-39.6$ & Double & 18 & (3) \\
\hline J1605.3+3249 (RBS 1556) & $\ldots$ & $\ldots$ & $\ldots$ & $353_{-48}^{+19}$ & $4.7_{-0.6}^{+0.00}$ & $\ldots$ & $\ldots$ & $<1.4$ & (4) \\
\hline $\mathrm{J} 1856.5-3754$ & 7.06 & 2.98 & 2.9 & $\ldots$ & $\ldots$ & $9.6-15.2$ & Single & 1.2 & (5) \\
\hline J2143.0+0654 (RBS 1774) & 9.43 & 4.1 & 4.0 & $326_{-79}^{+56}$ & $4.3_{-1.0}^{+0.7}$ & $33.2-41.8$ & Single & 4 & $(6-7)$ \\
\hline PSR J0726-2612 & 3.44 & 29.3 & 6.4 & $390_{-20}^{+10}$ & $5.2_{-0.3}^{+0.1}$ & $1.1-3.0$ & Double & 30 & (8) \\
\hline
\end{tabular}

Notes. $B_{\mathrm{p}}$ and $B_{\mathrm{cyc}}$ are the magnetic field at the poles evaluated from the timing parameter and from the cyclotron energy, respectively. $E_{\mathrm{cyc}}$ values are taken from Yoneyama et al. (2019), while $L_{X}$ values from Viganò et al. (2013).

References. (1) Haberl et al. (2004); (2) Hambaryan et al. (2017); (3) Hambaryan et al. (2011); (4) Pires et al. (2019); (5) Tiengo \& Mereghetti (2007); (6) Zane et al. (2005); (7) Mignani et al. (2011); (8) this paper.
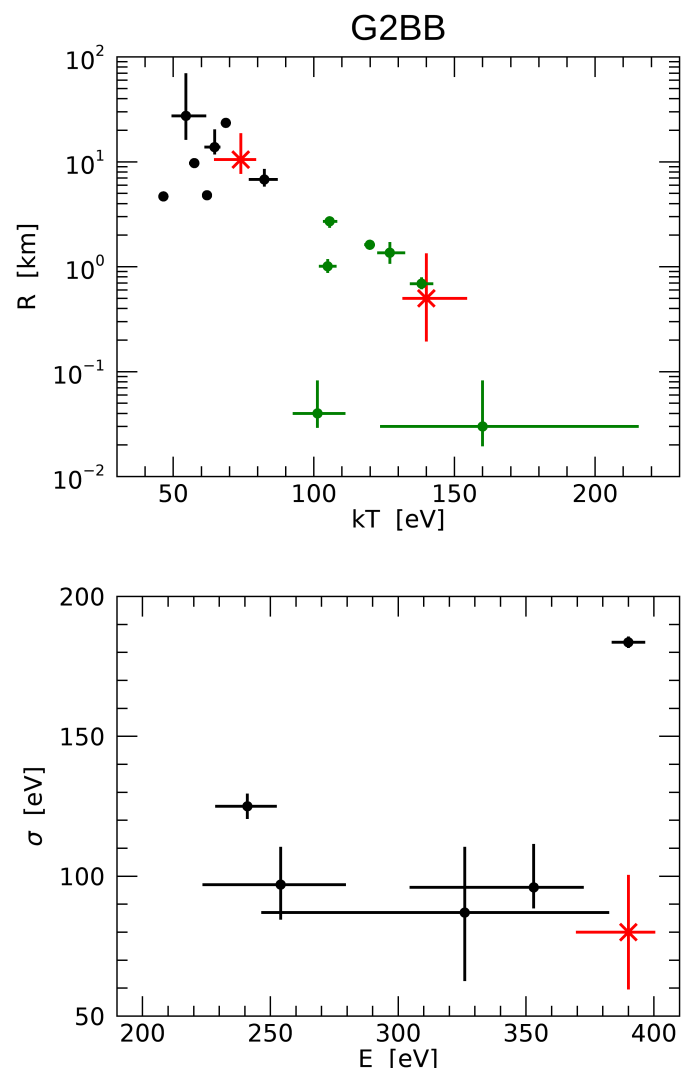

Fig. 8. Comparison of the spectral parameters of the XDINSs (from Yoneyama et al. 2019) and PSR J0726-2612 (red cross) obtained with two blackbodies and a Gaussian absorption line model (G2BB). Upper panel: blackbody radii (black: cold; green: hot) for the XDINSs and for PSR J0726-2612 (red cross). Lower panel: line width vs. line centroid energy (black dots are for XDINSs and the red cross for PSR J0726-2612).

J0720.4-3125, Hambaryan et al. 2017) show double-peaked profiles similar to PSR J0726-2612, although with smaller pulsed fractions (18\% and $11 \%$, respectively). The remarkable difference between PSR J0726-2612 and these two XDINSs is the presence of radio emission in the former. Here we discuss the pos-

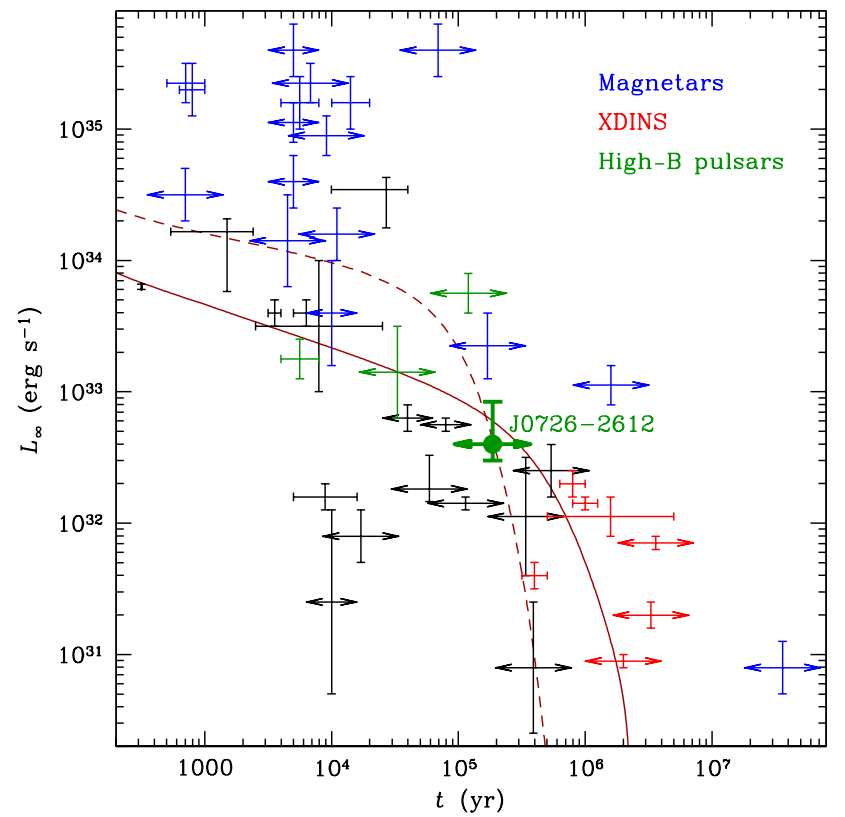

Fig. 9. Thermal luminosities vs. ages of isolated neutron stars. The same color coding as in Fig. 1 is used (in addition to PSR J0726-2612, the three High- $B$ pulsars are J1119-6127, J1718-3718 and J1819-1458). The solid and dashed lines are the theoretical cooling curves of a neutron star with mass $M=1.2 M_{\odot}$ and the dipole magnetic field of PSR J0726-2612 $\left(B_{\mathrm{p}}=6 \times 10^{13} \mathrm{G}\right)$, with the heat blanketing outer envelope composed either of iron (solid line) or of accreted light elements (dashed line).

sibility that this is due to an unfavorable orientation of their radio beam. Based on the radio beaming fraction of long-period pulsars, Kondratiev et al. (2009) estimated that a much larger number of XDINSs ( 40) would need to be observed to detect one with the radio beam crossing our line of sight.

We marked in Fig. 10 the values of the angles $\xi$ and $\chi$ estimated for RX J1308.6+2127 and RX J0720.4-3125 by Hambaryan et al. $(2011,2017)$. They imply that these two pulsars are nearly orthogonal rotators $\left(\xi \approx 90^{\circ}\right)$ seen with a large impact parameter $\eta=|\chi-\xi| \approx 45^{\circ}$. With the usual assumption that the radio beam coincides with, or is close to, the 


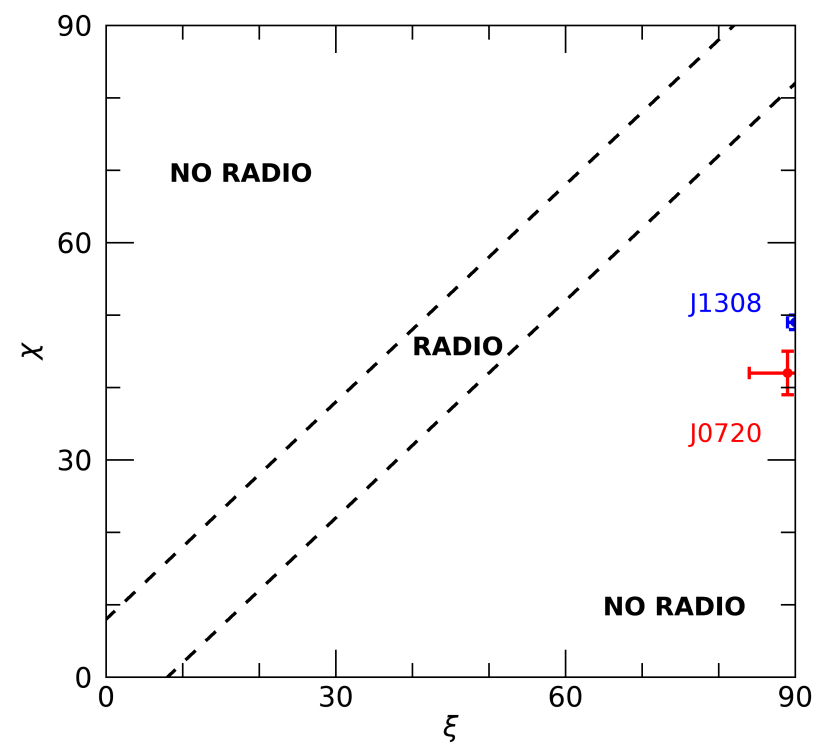

Fig. 10. Visibility of a radio beam with aperture of $\sim 8^{\circ}$ as a function of the $\xi$ and $\chi$ angles. The estimated positions for RX J1308.6+ 2127 (Hambaryan et al. 2011, blue dot) and RX J0720.4-3125 (Hambaryan et al. 2017, red dot) are shown.

magnetic dipole axis, such a large impact parameter can naturally account for the fact that their radio emission is not visible from the Earth. As an example, the dashed lines in Fig. 10 indicate the region where $\xi \approx \chi$ for which a radio beam with an aperture of $\sim 8^{\circ}$ would be visible. Contrary to the two XDINSs, PSR J0726-2612 should lie inside this region. Our atmosphere model used to compute the pulse profiles of Fig. 7 predicts that the radio pulse appearing when the magnetic axis is in the plane defined by the line of sight and rotation axis is in phase with one of the two minima of the X-ray profile. Considering the current relative error in the radio and X-ray phase alignment (see Fig. 2), this possibility cannot be excluded.

\section{Conclusions}

Our analysis of XMM-Newton data of the slow, highly magnetized radio pulsar PSR J0726-2612 reveals the presence of a broad absorption line in its soft thermal spectrum, with parameters similar to those of the lines seen in most of the XDINSs. The X-ray pulse profile of PSR J0726-2612 is double-peaked and moderately energy-dependent. These findings reinforce the similarity between this radio pulsar and the XDINSs. Assuming a distance of $1 \mathrm{kpc}$, the luminosity of PSR J0726-2612 is $L_{\infty}=\left(4.0_{-1.0}^{+4.4}\right) \times 10^{32} \mathrm{erg} \mathrm{s}^{-1}$. This is greater than its spin-down luminosity, as for the XDINSs (see Table 4), but is in reasonable agreement with the expected thermal luminosity of a $\sim 200 \mathrm{kyr}-$ old pulsar (see Fig. 9).

More observations are needed to reduce the uncertainty in the radio and X-ray phase alignment and better constrain the geometry of PSR J0726-2612. This could help to decipher whether or not the detection of radio emission in this pulsar, and not in the XDINSs with a similar double-peaked X-ray pulse profile, is due only to orientation effects.

Acknowledgements. We are grateful to an anonymous referee for constructive suggestions. We acknowledge financial contribution from the agreement ASIINAF n.2017-14-H.0. Part of this work has been funded using resources from the research grant "iPeska" (P.I. Andrea Possenti) funded under the INAF national call Prin-SKA/CTA approved with the Presidential Decree 70/2016. This work is based on observations obtained with XMM-Newton, an European Space Agency (ESA) science mission with instruments and contributions directly funded by ESA Member States and NASA. The work of A.Y.P. was supported by DFG and RFBR within the research project 19-52-12013. The work of V.S. was supported by Deutsche Forschungsgemeinschaft (DFG, (grant WE 1312/51-1)) and by the subsidy allocated to Kazan Federal University for the state assignment in the sphere of scientific activities (3.9780.2017/8.9).

\section{References}

Baldo, M., \& Schulze, H.-J. 2007, Phys. Rev. C, 75, 025802

Baring, M. G., \& Harding, A. K. 1998, ApJ, 507, L55

Baring, M. G., \& Harding, A. K. 2001, ApJ, 547, 929

Bhattacharya, D., Wijers, R. A. M. J., Hartman, J. W., \& Verbunt, F. 1992, A\&A, 254, 198

Burgay, M., Joshi, B. C., D’Amico, N., et al. 2006, MNRAS, 368, 283

Colpi, M., Geppert, U., \& Page, D. 2000, ApJ, 529, L29

Ding, D., Rios, A., Dussan, H., et al. 2016, Phys. Rev. C, 94, 025802

Haberl, F. 2007, Ap\&SS, 308, 181

Haberl, F., Motch, C., Zavlin, V. E., et al. 2004, A\&A, 424, 635

Hambaryan, V., Suleimanov, V., Schwope, A. D., et al. 2011, A\&A, 534, A74

Hambaryan, V., Suleimanov, V., Haberl, F., et al. 2017, A\&A, 601, A108

He, C., Ng, C.-Y., \& Kaspi, V. M. 2013, ApJ, 768, 64

Heyl, J. S., \& Kulkarni, S. R. 1998, ApJ, 506, L61

Ho, W. C. G. 2014, IAU Symp., 302, 435

Ho, W. C. G., Potekhin, A. Y., \& Chabrier, G. 2008, ApJS, 178, 102

Ho, W. C. G., Elshamouty, K. G., Heinke, C. O., \& Potekhin, A. Y. 2015, Phys. Rev. C, 91, 015806

Hobbs, G., Lorimer, D. R., Lyne, A. G., \& Kramer, M. 2005, MNRAS, 360, 974 Igoshev, A. P., Popov, S. B., \& Turolla, R. 2014, Astron. Nachr., 335, 262

Kalberla, P. M. W., Burton, W. B., Hartmann, D., et al. 2005, A\&A, 440, 775

Kaplan, D. L. 2008, AIP Conf. Ser., 968, 129

Kaspi, V. M. 2010, Proc. Natl. Acad. Sci., 107, 7147

Kaspi, V. M., \& Beloborodov, A. M. 2017, ARA\&A, 55, 261

Kondratiev, V. I., McLaughlin, M. A., Lorimer, D. R., et al. 2009, ApJ, 702, 692

Malofeev, V. M., Malov, O. I., Teplykh, D. A., Tyul'Bashev, S. A., \& Tyul'Basheva, G. E. 2005, Astron. Rep., 49, 242

Malofeev, V. M., Malov, O. I., Teplykh, D. A., et al. 2006, ATel, 798

Manchester, R. N., Hobbs, G. B., Teoh, A., \& Hobbs, M. 2005, VizieR Online Data Catalog, VII/7245

Margueron, J., Sagawa, H., \& Hagino, K. 2008, Phys. Rev. C, 77, 054309

Mereghetti, S. 2011, Astrophys. Space Sci. Proc., 21, 345

Mereghetti, S., Pons, J. A., \& Melatos, A. 2015, Space Sci. Rev., 191, 315

Mignani, R. P., Zane, S., Turolla, R., et al. 2011, A\&A, 530, A39

Pavlov, G. G., Shibanov, Y. A., Ventura, J., \& Zavlin, V. E. 1994, A\&A, 289, 837

Pavlov, G. G., Shibanov, Y. A., Zavlin, V. E., \& Meyer, R. D. 1995, in NATO

Advanced Science Institutes (ASI) Series C, eds. M. A. Alpar, U. Kiziloglu, \& J. van Paradijs, 450, 71

Pearson, J. M., Chamel, N., Potekhin, A. Y., et al. 2018, MNRAS, 481, 2994

Pires, A. M., Schwope, A. D., Haberl, F., et al. 2019, A\&A, 623, A73

Popov, S. B., Colpi, M., Prokhorov, M. E., Treves, A., \& Turolla, R. 2003, A\&A, 406, 111

Popov, S. B., Turolla, R., Prokhorov, M. E., Colpi, M., \& Treves, A. 2005, Ap\&SS, 299, 117

Potekhin, A. Y., \& Chabrier, G. 2018, A\&A, 609, A74

Potekhin, A. Y., Chabrier, G., \& Ho, W. C. G. 2014, A\&A, 572, A69

Rigoselli, M., Mereghetti, S., Turolla, R., et al. 2019, ApJ, 872, 15

Speagle, J. S., Kaplan, D. L., \& van Kerkwijk, M. H. 2011, ApJ, 743, 183

Strüder, L., Briel, U., Dennerl, K., et al. 2001, A\&A, 365, L18

Suleimanov, V., Potekhin, A. Y., \& Werner, K. 2009, A\&A, 500, 891

Tan, C. M., Bassa, C. G., Cooper, S., et al. 2018, ApJ, 866, 54

Tetzlaff, N., Eisenbeiss, T., Neuhäuser, R., \& Hohle, M. M. 2011, MNRAS, 417, 617

Tiengo, A., \& Mereghetti, S. 2007, ApJ, 657, L101

Turner, M. J. L., Abbey, A., Arnaud, M., et al. 2001, A\&A, 365, L27

Turolla, R. 2009, W. Becker, 357, 141

Turolla, R., \& Nobili, L. 2013, ApJ, 768, 147

Turolla, R., Zane, S., \& Watts, A. L. 2015, Rep. Prog. Phys., 78, 116901

Viganò, D., Rea, N., Pons, J. A., et al. 2013, MNRAS, 434, 123

Viganò, D., Perna, R., Rea, N., \& Pons, J. A. 2014, MNRAS, 443, 31

Walter, F. M., Eisenbeiß, T., Lattimer, J. M., et al. 2010, ApJ, 724, 669

Wilms, J., Allen, A., \& McCray, R. 2000, ApJ, 542, 914

Yao, J. M., Manchester, R. N., \& Wang, N. 2017, ApJ, 835, 29

Yoneyama, T., Hayashida, K., Nakajima, H., \& Matsumoto, H. 2019, PASJ, 71, 17

Zane, S., Cropper, M., Turolla, R., et al. 2005, ApJ, 627, 397 